\title{
FIXAÇÃO PERCUTÂNEA NO TRATAMENTO DE FRATURAS TORACOLOMBARES: RESULTADOS PRELIMINARES
}

\author{
PERCUTANEOUS FIXATION IN THE TREATMENT OF THORACOLUMBAR FRACTURES: \\ PRELIMINARY RESULTS
}

\section{FIJACIÓN PERCUTÁNEA EN EL TRATAMIENTO DE LAS FRACTURAS TORACOLUMBARES: RESULTADOS PRELIMINARES}

Anderson Gomes Marin, Carlos Fernando Pereira da Silva Herrero', Marcello Henrique Nogueira Barbosa², Helton Luiz Aparecido Defino ${ }^{1}$

\begin{abstract}
RESUMO
Objetivo: Avaliar os resultados preliminares do tratamento cirúrgico de pacientes portadores de fraturas toracolombares da coluna vertebral por meio de fixação percutânea. Métodos: Foram avaliados 10 pacientes com diagnóstico de fratura toracolombar da coluna vertebral. Todos os pacientes foram tratados por meio de técnica minimamente invasiva, com fixação por via posterior sem artrodese. Os parâmetros radiológicos relacionados para a avaliação dos pacientes foram: medida da cifose segmentar, encunhamento da vértebra fraturada e complicações relacionadas à soltura ou quebra dos implantes. Resultados: A análise clínica preliminar foi realizada por meio da avaliação subjetiva dos resultados da cirurgia e todos os pacientes mostraram-se satisfeitos com o procedimento cirúrgico. A avaliação radiográfica não mostrou sinais de mau posicionamento dos implantes, soltura ou quebra dos parafusos, assim como não houve falha mecânica no instrumental. Conclusão: A utilização da fixação percutânea minimamente invasiva para o tratamento das fraturas toracolombares da coluna vertebral representa uma boa opção de tratamento, apresentando recuperação mais rápida, com resultados clínicos e radiográficos comparáveis às técnicas convencionais.
\end{abstract}

Descritores: Coluna vertebral; Traumatismos da coluna vertebral; Fraturas da coluna vertebral.

\begin{abstract}
Objective: To evaluate the preliminary results of the surgical treatment of patients with thoracolumbar fractures of the spine using percutaneous fixation. Methods: We evaluated 10 patients with diagnosis of thoracolumbar fractures of the spine. All patients were treated with minimally invasive technique via posterior fixation without arthrodesis. The radiological parameters related to the evaluation of patients: measurement of segmental kyphosis, wedging of the fractured vertebra and the complications related to loosening or breakage of implants. Results: A preliminary clinical analysis was performed by means of subjective evaluation of the surgery outcomes and all patients were satisfied with the surgical procedure. Radiographic evaluation showed no signs of implant malposition, loosening or breakage of the screws, as well as no mechanical failure in the instrumental. Conclusion: The minimally invasive percutaneous fixation for the treatment of thoracolumbar fractures of the spine is a good treatment option, with a quicker recovery, and clinical and radiographic results comparable to conventional techniques.
\end{abstract}

Keywords: Spine; Spinal injuries; Spinal fractures.

RESUMEN

Objetivo: Evaluar los resultados preliminares del tratamiento quirúrgico de los pacientes con fracturas de columna toracolumbar mediante fijación percutánea. Métodos: Se evaluaron 10 pacientes con diagnóstico de fractura toracolumbar de la columna vertebral. Todos los pacientes fueron tratados mediante la técnica mínimamente invasiva a través de la fijación posterior sin artrodesis. Los parámetros radiológicos relacionados con la evaluación de los pacientes fueron: medición de la cifosis segmentaria, acuñamiento de la vértebra fracturada y las complicaciones relacionadas con el aflojamiento o rotura de los implantes. Resultados: La valoración clínica preliminar se realizó por medio de la evaluación subjetiva de los resultados de la cirugía y todos los pacientes se mostraron satisfechos con el procedimiento quirúrgico. La evaluación radiográfica no mostró signos de mala ubicación de los implantes, tampoco de aflojamiento del implante o rotura de los tornillos. Asimismo no hubo fallo mecánico del instrumental. Conclusión: El uso de la fijación percutánea mínimamente invasiva para el tratamiento de las fracturas toracolumbares de la columna vertebral es una buena opción de tratamiento, con recuperación más rápida, con resultados clínicos y radiográficos comparables a las técnicas convencionales.

Descriptores: Columna vertebral; Fracturas de la columna vertebral; Traumatismos vertebrales.

\section{INTRODUÇÃO}

As fraturas toracolombares da coluna vertebral ainda são fontes de controvérsias no que diz respeito a critérios de instabilidade e algoritmos de tratamento. ${ }^{1}$ Questões como a necessidade da abordagem anterior nas lesões com fragmentação do corpo vertebral e a realização da artrodese após a fixação posterior ainda permanecem.

Dentre as opções de tratamento cirúrgico, não existe aborda- gem cirúrgica padronizada para o tratamento das fraturas da coluna. As opções atuais de tratamento cirúrgico incluem as abordagens abertas convencionais e a utilização de instrumentação com artrodese. Nos pacientes traumatizados, as técnicas convencionais podem estar associadas à morbidade significativa, como a perda sanguinea e o aumento da taxa de infecção. ${ }^{1-5}$

Com a difusão das técnicas minimamente invasiva, uma nova

\footnotetext{
1. Departamento de Biomecânica, Medicina e Reabilitação do Aparelho Locomotor do Hospital das Clínicas da Faculdade de Medicina de Ribeirão Preto - USP - Ribeirão Preto, SP, Brasil.
} 2. Divisão de Radiologia da Faculdade de Medicina de Ribeirão Preto - USP - Ribeirão Preto, SP, Brasil.

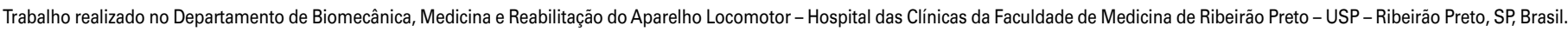
Correspondência: Helton L.A. Defino, AV Bandeirantes, 3900, $11^{\circ}$ andar. 14048-900. Ribeirão Preto. SP, Brasil. hladefin @ fmrp.usp.br 
discussão veio à tona: a necessidade ou não de manter um paciente sob imobilização e restrições de mobilização sendo que um procedimento com pouca morbidade poderia ser utilizado e dar ao indivíduo uma melhor qualidade de vida durante sua recuperação.

Neste contexto, alguns estudos foram feitos para verificar se as fixações minimamente invasivas por via posterior para as lesões que antes poderiam ser tratadas conservadoramente tratariam o benefício esperado sem trazer a morbidade dos grandes acessos posteriores. ${ }^{6-8}$

O objetivo deste estudo foi avaliar os resultados preliminares do tratamento cirúrgico de pacientes portadores de fraturas toracolombares da coluna vertebral por meio de fixação percutânea.

\section{MATERIAL E MÉTODOS}

Foram avaliados 10 pacientes com o diagnóstico de fratura toracolombar da coluna vertebral que deram entrada na Unidade de Emergência da Faculdade de Medicina de Ribeirão Preto da Universidade de São Paulo entre fevereiro e outubro de 2010. Oito pacientes eram do sexo masculino e dois do sexo feminino e a idade variou de 16 a 42 anos (média de 27,3 anos). Os dados gerais dos pacientes estão ilustrados na Tabela 1.

A causa da fratura foi acidente de trânsito em sete pacientes (70\%) e queda de altura em três pacientes (30\%). A localização da fratura era um em T12 (10\%), três em L1 (30\%), quatro em L2 (40\%) e dois em L4 (20\%). Todas as fraturas foram classificadas de acordo com a classificação de Magerl et al. ${ }^{9}$ : três do tipo A (30\%), três do tipo B (30\%) e quatro do tipo C (40\%). (Tabela 1$)$

Todos os pacientes foram tratados por meio de técnica minimamente invasiva com fixação via posterior sem artrodese. Em cinco pacientes (50\%) foi utilizado o sistema Sextant ${ }^{\circledR}$ (Medtronic) (Figura 1) e em cinco (50\%) foi utilizada a inserção de parafusos poliaxiais Basis ${ }^{\circledR}$ (Medtronic) (Figura 2) por meio de incisão trans-fascial, após incisão mediana da pele. Somente um paciente (paciente 9 da Tabela 1) necessitou de abordagem complementar (descompressão e artrodese via anterior) devido à presença de déficit neurológico.

No período pós-operatório os pacientes não utilizaram órtese e não houve, relacionadas à coluna vertebral, restrições para deambular ou na reabilitação, que era feita de acordo com as condições gerais do paciente e com a resposta à dor.

Os parâmetros radiológicos relacionados para a avaliação dos pacientes foram: a medida da cifose segmentar, encunhamento da vértebra fraturada e as complicações relacionadas à soltura ou quebra dos implantes. Os parâmetros foram avaliados no préoperatório, pós-operatório imediato.

Tabela 1. Dados dos pacientes avaliados no estudo.

\begin{tabular}{|c|c|c|c|c|c|c|c|}
\hline Paciente & Idade & Sexo & MT & Topografia & Classificação & Lesão Neurológica & Outras lesões \\
\hline 1 & 37 & $\mathrm{M}$ & Moto & $\mathrm{L} 1$ & A2.1 & - & - \\
\hline 2 & 22 & $\mathrm{M}$ & Moto & $\mathrm{L} 1$ & B1.2 & - & - \\
\hline 3 & 16 & $\mathrm{~F}$ & Queda $5 \mathrm{~m}$ & L2/L3 & C1.1 & - & Fratura calcâneo \\
\hline 4 & 34 & $\mathrm{M}$ & Queda 4m & $\mathrm{L} 2$ & B1.2 & - & Fratura talus \\
\hline 5 & 42 & $\mathrm{M}$ & Carro & $\mathrm{L} 2$ & C1.1 & - & - \\
\hline 6 & 36 & $\mathrm{M}$ & Queda 3m & $\mathrm{L} 1$ & A1.2 & - & - \\
\hline 7 & 31 & $\mathrm{M}$ & Moto & L4 & C1.1 & - & Fratura metatarso \\
\hline 8 & 19 & $\mathrm{M}$ & Moto & T12 & B1.1 & - & - \\
\hline 9 & 17 & $\mathrm{M}$ & Moto & $\mathrm{L} 4$ & A3.2 & Raiz L4 & Fratura tíbial \\
\hline 10 & 19 & $F$ & Moto & $\mathrm{L} 2$ & C1.1 & - & Amputação da perna \\
\hline
\end{tabular}

Legenda: M: masculino; F: feminino; MT: mecanismo de trauma; L: Iombar; T: torácica.

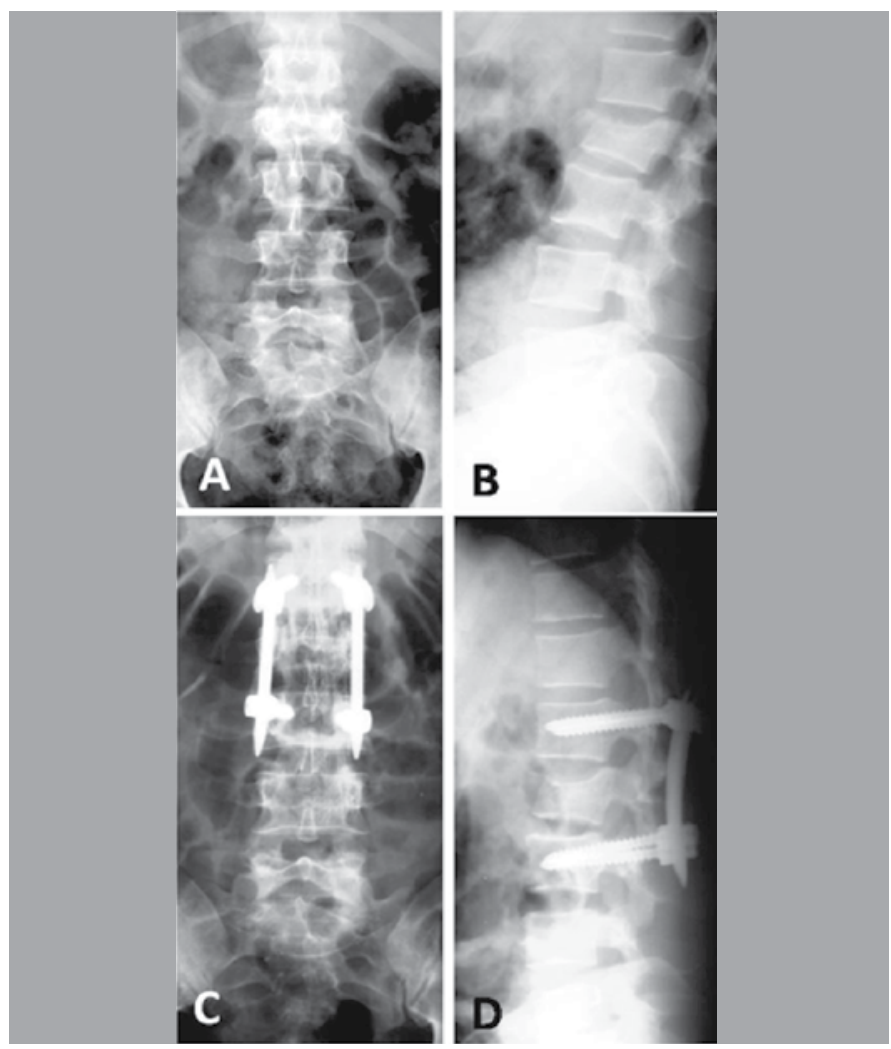

Figura 1. Paciente de 34 anos de idade, vítima de acidente de trânsito, com diagnóstico de fratura de L2. A e B: Incidência radiográfica anteroposterior e perfil evidenciando a fratura de L2. C e D: dois meses após fixação e estabilização com técnica minimamente invasiva, Sextant ${ }^{\circledR}$ (Medtronic).
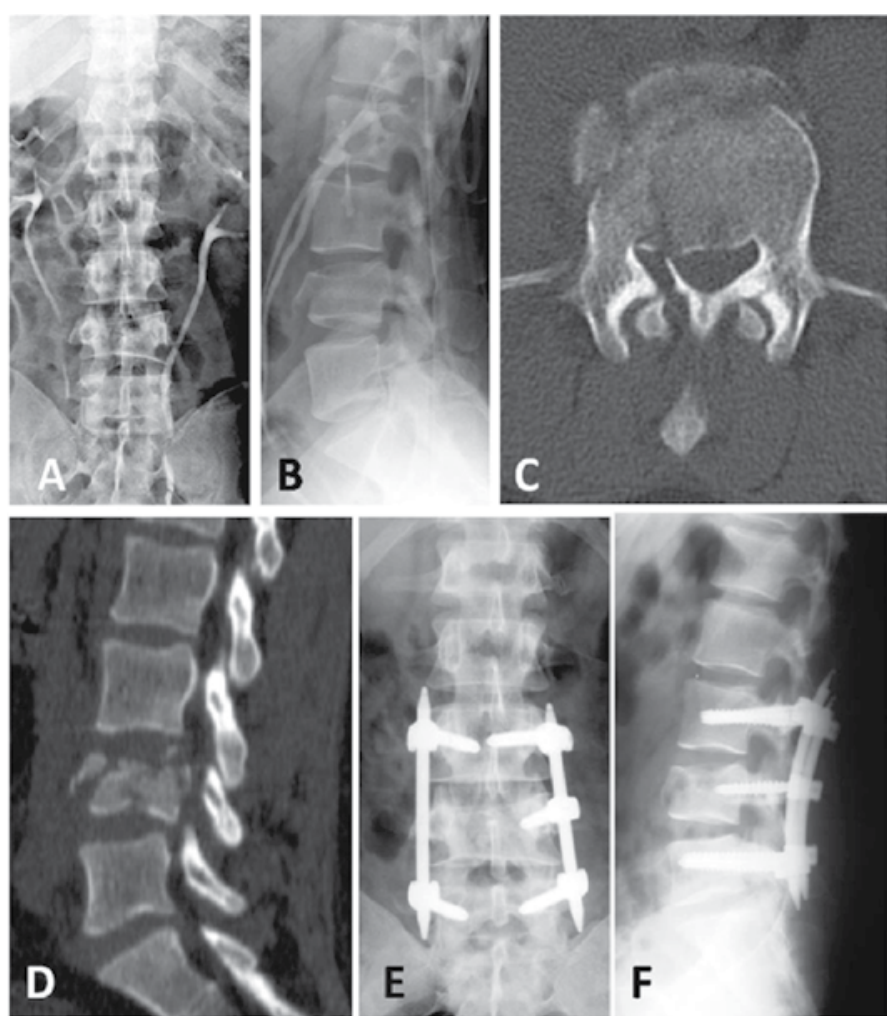

Figura 2. Paciente de 31 anos de idade, vítima de acidente de trânsito, com diagnóstico de fratura de $L 4$. A e B: Incidência radiográfica anteroposterior e perfil evidenciando a fratura de $L 4$. C e D: tomografia computadorizada pré-operatória. E e F: dois meses após fixação e estabilização com técnica minimamente invasiva, Sextant ${ }^{\circledR}$ (Medtronic). 


\section{RESULTADOS}

A avaliação clínica preliminar foi realizada por meio da avaliação subjetiva dos resultados da cirurgia e todos os pacientes mostraram-se satisfeitos com o procedimento cirúrgico.

A avaliação radiográfica não mostrou sinais de mau posicionamento dos implantes, soltura ou quebra dos parafusos. Assim como não houve falha mecânica no instrumental. (Tabela 1)

A transição toracolombar (T12-L1) e o segmento L2 foram os mais acometidos pelas lesões (40\%). Somente um paciente apresentava lesão neurológica (paciente 9 da Tabela 1) sendo que a função foi parcialmente restabelecida após a descompressão e totalmente restabelecida após três meses da lesão. As lesões dos membros inferiores estiveram presentes em 50\% dos pacientes, entretanto lesões que colocassem em risco a vida do indivíduo não foram observadas.

\section{DISCUSSÃO}

As técnicas minimamente invasivas de fixação da coluna vertebral têm mostrado grande avanço. Inúmeros implantes já estão disponíveis para fixação de um ou múltiplos segmentos vertebrais. Diversas têm sido as indicações de sua utilização, desde o tratamento de fraturas até o tratamento de deformidades.

A razão para a aplicação das técnicas minimamente invasivas no tratamento das fraturas da coluna vertebral é reduzir a incidência de complicações relacionadas com a abordagem convencional aberta como a desnervação da musculatura paravertebral, aumento da pressão intramuscular, isquemia muscular, dor e perda de função., ${ }^{4,7}$

A abordagem minimamente invasiva posterior tem sido apontada como uma opção no tratamento das fraturas toracolombares. ${ }^{6-8}$ Brodano et al. ${ }^{10}$ operaram 25 pacientes com fraturas do tipo A e B segundo a classificação de Magerl et al. ${ }^{9}$ e apresentaram bons resultados clínicos. Assim, sugerem uma instrumentação curta para o tratamento das fraturas do tipo A como uma alternativa ao tratamento convencional. ${ }^{10}$
Em um estudo, Wang et al. ${ }^{11}$ compararam dois grupos de pacientes com fraturas toracolombares do tipo explosão. Um grupo de pacientes foi tratado por meio de artrodese instrumentada, enquanto o outro grupo de pacientes foi submetido apenas à fixação, sem artrodese. No entanto, não foi encontrada diferença estatística significativa na avaliação tardia entre os dois grupos no que diz respeito aos parâmetros radiográficos e clínicos. ${ }^{11}$ Esta informação corrobora com a utilização da técnica minimamente invasiva em nosso estudo.

Alguns estudos apontaram uma perda da correção e encunhamento da vértebra fraturada. ${ }^{6,12,13}$ Estamos de acordo com Palmisani et al. ${ }^{7}$ que o grande responsável por essa perda seria a utilização de parafusos poliaxiais e que, por essa razão, haverá espaço para a utilização de parafusos monoaxiais.

Acreditamos que a maior crítica que poderia existir para a fixação das fraturas toracolombares por meio de técnica minimamente invasiva seria a utilização de implantes percutâneos longos no tratamento das fraturas do tipo explosão, uma vez que haveria um sacrifício adicional de segmentos de mobilidade. Assim, esse tipo de instrumentação não foi utilizado em nosso estudo (paciente 9 da Tabela 1), sendo optado por técnica minimamente de abordagem anterior.

\section{CONCLUSÃO}

A utilização da fixação percutânea minimamente invasiva para o tratamento das fraturas toracolombares da coluna vertebral representa uma boa opção de tratamento, apresentando uma recuperação mais rápida, com resultados clínicos e radiográficos comparáveis às técnicas convencionais.

Todos os autores declaram não haver nenhum potencial conflito de interesses referente a este artigo.

\section{REFERÊNCIAS}

1. Verlaan JJ, Diekerhof CH, Buskens E, van der Tweel I, Verbout AJ, Dhert WJ, et al. Surgical treatment of traumatic fractures of the thoracic and lumbar spine: a systematic review of the literature on techniques, complications, and outcome. Spine (Phila Pa 1976). 2004;29(7):803-14

2. Gejo R, Kawaguchi Y, Kondoh T, Tabuchi E, Matsui H, Torii K, et al. Magnetic resonance imaging and histologic evidence of postoperative back muscle injury in rats. Spine (Phila Pa 1976). 2000;25(8):941-6

3. KawaguchiY, Yabuki S, Styf J, Olmarker K, Rydevik B, Matsui H, et al. Back muscle injury after posterior lumbar spine surgery. Topographic evaluation of intramuscular pressure and blood flow in the porcine back muscle during surgery. Spine (Phila Pa 1976). 1996;21(22):2683-8.

4. Kawaguchi $Y$, Matsui $H$, Tsuji $H$. Back muscle injury after posterior lumbar spine surgery. $A$ histologic and enzymatic analysis. Spine (Phila Pa 1976). 1996;21(8):941-4.

5. Kawaguchi Y, Matsui H, Gejo R, Tsuji H. Preventive measures of back muscle injury after posterior lumbar spine surgery in rats. Spine (Phila Pa 1976). 1998;23(21):2282-7.

6. Logroscino CA, Proietti L, Tamburrelli FC. Minimally invasive spine stabilisation with long implants. Eur Spine J. 2009;18(Suppl 1):75-81.

7. Palmisani M, Gasbarrini A, Brodano GB, De lure F, Cappuccio M, Boriani L, et al. Minimally invasive percutaneous fixation in the treatment of thoracic and lumbar spine fractures.
Eur Spine J. 2009:18(Suppl 1):71-4

8. Rampersaud YR, Annand N, Dekutoski MB. Use of minimally invasive surgical techniques in the management of thoracolumbar trauma: current concepts. Spine (Phila Pa 1976). 2006;31(Suppl 11):S96-102.

9. Magerl F, Aebi M, Gertzbein SD, Harms J, Nazarian S. A comprehensive classification of thoracic and lumbar injuries. Eur Spine J. 1994;3(4):184-201.

10. Brodano GB, De lure F, Cappuccio M, Palmisani M, Boriani L, Gasbarrini A, et al. Osteosintese con tecnica percutanea mininvasiva nel trattamento delle fratture vertebrali toraciche e lombari. Esperienza preliminare. GIOT. 2007;33:78-85.

11. Wang ST, Ma HL, Liu CL, Yu WK, Chang MC, Chen TH. Is fusion necessary for surgically treated burst fractures of the thoracolumbar and lumbar spine?: a prospective, randomized study. Spine (Phila Pa 1976). 2006;31(23):2646-52.

12. Altay M, Ozkurt B, Aktekin CN, Ozturk AM, Dogan O, Tabak AY. Treatment of unstable thoracolumbar junction burst fractures with short- or long-segment posterior fixation in magerl type a fractures. Eur Spine J. 2007;16(8):1145-55.

13. Kuklo TR, Polly DW, Owens BD, Zeidman SM, Chang AS, Klemme WR. Measurement of thoracic and lumbar fracture kyphosis: evaluation of intraobserver, interobserver, and technique variability. Spine (Phila Pa 1976). 2001;26(1):61-5. 